DECIGO pathfinder

This article has been downloaded from IOPscience. Please scroll down to see the full text article.

2009 Class. Quantum Grav. 26094019

(http://iopscience.iop.org/0264-9381/26/9/094019)

View the table of contents for this issue, or go to the journal homepage for more

Download details:

IP Address: 194.94.224.254

The article was downloaded on 15/06/2010 at $11: 37$

Please note that terms and conditions apply. 


\title{
DECIGO pathfinder
}

\author{
Masaki Ando ${ }^{1,58}$, Seiji Kawamura ${ }^{2}$, Shuichi Sato ${ }^{3}$, Takashi Nakamura ${ }^{4}$, \\ Kimio Tsubono ${ }^{1}$, Akito Araya ${ }^{5}$, Ikkoh Funaki ${ }^{6}$, Kunihito Ioka ${ }^{7}$, \\ Nobuyuki Kanda $^{8}$, Shigenori Moriwaki ${ }^{9}$, Mitsuru Musha ${ }^{10}$, \\ Kazuhiro Nakazawa $^{1}$, Kenji Numata ${ }^{11}$, Shin-ichiro Sakai ${ }^{6}$, Naoki Seto ${ }^{2}$, \\ Takeshi Takashima $^{6}$, Takahiro Tanaka ${ }^{12}$, Kazuhiro Agatsuma ${ }^{1}$, \\ Koh-suke Aoyanagi $^{13}$, Koji Arai $^{2}$, Hideki Asada ${ }^{14}$, Yoichi Aso ${ }^{15}$, \\ Takeshi Chiba $^{16}$, Toshikazu Ebisuzaki ${ }^{17}$, Yumiko Ejiri ${ }^{18}$, \\ Motohiro Enoki $^{19}$, Yoshiharu Eriguchi ${ }^{20}$, Masa-Katsu Fujimoto ${ }^{2}$, \\ Ryuichi Fujita $^{21}$, Mitsuhiro Fukushima ${ }^{2}$, Toshifumi Futamase ${ }^{22}$, \\ Katsuhiko Ganzu ${ }^{4}$, Tomohiro Harada ${ }^{23}$, Tatsuaki Hashimoto ${ }^{6}$, \\ Kazuhiro Hayama $^{24}$, Wataru Hikida ${ }^{25}$, Yoshiaki Himemoto ${ }^{26}$, \\ Hisashi Hirabayashi $^{27}$, Takashi Hiramatsu ${ }^{28}$, Feng-Lei Hong ${ }^{29}$, \\ Hideyuki Horisawa $^{30}$, Mizuhiko Hosokawa ${ }^{31}$, Kiyotomo Ichiki ${ }^{1}$, \\ Takeshi Ikegami $^{29}$, Kaiki T Inoue ${ }^{32}$, Koji Ishidoshiro ${ }^{1}$, Hideki Ishihara ${ }^{8}$, \\ Takehiko Ishikawa $^{6}$, Hideharu Ishizaki ${ }^{2}$, Hiroyuki Ito ${ }^{31}$, Yousuke Itoh ${ }^{33}$, \\ Nobuki Kawashima $^{32}$, Fumiko Kawazoe ${ }^{34}$, Naoko Kishimoto ${ }^{6}$, \\ Kenta Kiuchi $^{13}$, Shiho Kobayashi ${ }^{35}$, Kazunori Kohri ${ }^{36}$, \\ Hiroyuki Koizumi $^{6}$, Yasufumi Kojima ${ }^{37}$, Keiko Kokeyama ${ }^{18}$, \\ Wataru@Kokuyama ${ }^{1}$, Kei Kotake ${ }^{2}$, Yoshihide Kozai ${ }^{38}$, Hideaki Kudoh ${ }^{1}$, \\ Hiroo Kunimori ${ }^{31}$, Hitoshi Kuninaka ${ }^{6}$, Kazuaki Kuroda ${ }^{28}$, \\ Kei-ichi Maeda $^{13}$, Hideo Matsuhara ${ }^{6}$, Yasushi Mino ${ }^{15}$, \\ Osamu Miyakawa $^{15}$, Shinji Miyoki $^{28}$, Mutsuko Y Morimoto ${ }^{6}$, \\ Tomoko Morioka ${ }^{1}$, Toshiyuki Morisawa ${ }^{4}$, Shinji Mukohyama ${ }^{39}$, \\ Shigeo Nagano ${ }^{31}$, Isao Naito ${ }^{40}$, Kouji Nakamura ${ }^{2}$, Hiroyuki Nakano ${ }^{41}$, \\ Kenichi Nakao $^{8}$, Shinichi Nakasuka ${ }^{42}$, Yoshinori Nakayama ${ }^{43}$, \\ Erina Nishida $^{18}$, Kazutaka Nishiyama ${ }^{6}$, Atsushi Nishizawa ${ }^{44}$, \\ Yoshito Niwa $^{44}$, Taiga Noumi ${ }^{42}$, Yoshiyuki Obuchi ${ }^{2}$, Masatake Ohashi ${ }^{28}$, \\ Naoko Ohishi $^{2}$, Masashi Ohkawa ${ }^{45}$, Norio Okada ${ }^{2}$, Kouji Onozato ${ }^{1}$, \\ Kenichi Oohara $^{45}$, Norichika Sago ${ }^{46}$, Motoyuki Saijo ${ }^{47}$, \\ Masaaki Sakagami $^{44}$, Shihori Sakata ${ }^{2}$, Misao Sasaki ${ }^{12}$, Takashi Sato ${ }^{45}$, \\ Masaru Shibata $^{20}$, Hisaaki Shinkai ${ }^{48}$, Kentaro Somiya ${ }^{15}$, \\ Hajime Sotani $^{49}$, Naoshi Sugiyama ${ }^{50}$, Yudai Suwa ${ }^{1}$, Rieko Suzuki ${ }^{18}$, \\ Hideyuki Tagoshi $^{25}$, Fuminobu Takahashi ${ }^{39}$, Kakeru Takahashi $^{1}$, \\ Keitaro Takahashi $^{12}$, Ryutaro Takahashi ${ }^{2}$, Ryuichi Takahashi ${ }^{50}$, \\ Tadayuki Takahashi $^{6}$, Hirotaka Takahashi ${ }^{51}$, Takamori Akiteru ${ }^{5}$, \\ Tadashi Takano $^{52}$, Keisuke Taniguchi ${ }^{33}$, Atsushi Taruya ${ }^{1}$, \\ Hiroyuki Tashiro ${ }^{4}$, Yasuo Torii ${ }^{2}$, Morio Toyoshima ${ }^{31}$, \\ Shinji Tsujikawa $^{53}$, Yoshiki Tsunesada ${ }^{54}$, Akitoshi Ueda ${ }^{2}$, \\ Ken-ichi Ueda $^{10}$, Masayoshi Utashima ${ }^{55}$, Yaka Wakabayashi ${ }^{18}$, \\ Hiroshi Yamakawa ${ }^{56}$, Kazuhiro Yamamoto ${ }^{34}$, Toshitaka Yamazaki ${ }^{2}$,
}




\section{Jun'ichi Yokoyama ${ }^{1}$, Chul-Moon Yoo $^{8}$, Shijun Yoshida ${ }^{22}$ and Taizoh Yoshino ${ }^{57}$}

${ }^{1}$ Department of Physics, The University of Tokyo, Tokyo 113-0033, Japan

2 National Astronomical Observatory Japan, Osawa 2-21-1, Tokyo 181-8588, Japan

${ }^{3}$ Faculty of Engineering, Hosei University, kajinocho, Tokyo 184-8584, Japan

${ }^{4}$ Department of Physics, Kyoto University, Kyoto 606-8502, Japan

${ }^{5}$ Earthquake Research Institute, The University of Tokyo, Tokyo 113-0032, Japan

${ }^{6}$ Institute of Space and Astronautical Science, Japan Aerospace Exploration Agency (JAXA), Kanagawa 229-8510, Japan

${ }^{7}$ Institute of Particle and Nuclear Studies, High Energy Accelerator Research Organization (KEK), Ibaraki 305-0801, Japan

${ }^{8}$ Department of Physics, Osaka City University, Osaka 558-8585, Japan

${ }^{9}$ Department of Advanced Materials Science, The University of Tokyo, Chiba 277-8561, Japan

${ }^{10}$ Institute for Laser Science, The University of Electro-Communications, Tokyo 182-8585, Japan 11 NASA Goddard Space Flight Center, Code 663, 8800 Greenbelt Rd, Greenbelt, MD 20771, USA

12 Yukawa Institute for Theoretical Physics, Kyoto University, Kyoto 606-8502, Japan

${ }^{13}$ Department of Physics, Waseda University, Tokyo 169-8555, Japan

${ }^{14}$ Department of Earth and Environmental Sciences, Hirosaki University, Aomori 036-8560, Japan

${ }_{15}$ California Institute of Technology, 1200 E California Blvd MC 18-34, Pasadena, CA 91125 , USA

${ }^{16}$ Nihon University, Setagaya, Tokyo 156-8550, Japan

17 RIKEN, 2-1 Hirosawa, Wako 351-0198, Japan

18 Ochanomizu University, 2-1-1, Tokyo 112-0012, Japan

${ }^{19}$ Faculty of Business Administration, Tokyo Keizai University, Tokyo 185-8502, Japan

${ }^{20}$ Department of Earth Science and Astronomy, The University of Tokyo, Tokyo 153-8902, Japan

21 Theoretical Physics, Raman Research Institute, Sir C V Raman Avenue, Sadashivanagar P O, Bangalore 560 080, India

22 Astronomical Institute, Tohoku University, Sendai 980-8578, Japan

${ }^{23}$ Department of Physics, Rikkyo University, Tokyo 171-8501, Japan

${ }^{24}$ University of Texas, 80 Fort Brown, Brownsville, 78520 TX, USA

25 Department of Earth and Space Science, Osaka University, Osaka 560-0043, Japan

${ }^{26}$ Center for Educational Assistance, Shibaura Institute of Technology, Saitama 337-8570, Japan

27 Space Educations Center, Japan Aerospace Exploration Agency (JAXA), Kanagawa 229-8510, Japan

${ }^{28}$ Institute for Cosmic Ray Research, The University of Tokyo, Chiba 277-8582, Japan

${ }^{29}$ National Institute of Advanced Industrial Science and Technology (AIST), Ibaraki 305-8563, Japan

${ }^{30}$ Department of Aeronautics and Astronautics, Tokai University, Kanagawa 259-1292, Japan

${ }^{31}$ National Institute of Information and Communications Technology (NICT), Tokyo 184-8795, Japan

${ }^{32}$ School of Science and Engineering, Kinki University, Osaka 577-8502, Japan

33 Department of Physics, University of Wisconsin-Milwaukee, 1900 East Kenwood Blvd, Milwaukee, WI 53211, USA

${ }^{34}$ Max-Planck-Institute for Gravitational Physics (Albert-Einstein-Institute), Callinstr 38 D-30167 Hannover, Germany

35 Astrophysics Research Institute, Liverpool John Moores University, Twelve Quays House, Egerton Wharf, Birkenhead L41 1LD, UK

36 Theoretical Astrophysics, Institute for Astronomy and Astrophysics, Eberhard Karls University of Tuebingen, 72076 Tuebingen, Germany

37 Graduate School of Science, Hiroshima University, Hiroshima 739-8526, Japan

38 Gunma Astronomical Observatory, Agatsuma-gun, Gunma 377-0702, Japan

${ }^{39}$ Institute for Physics and Mathematics of the Universe (IPMU), The University of Tokyo, Chiba 277-8568, Japan

${ }^{40}$ Numakage, Saitama-shi, Saitama 336-0027 Japan

${ }^{41}$ Rochester Institute of Technology, 78 Lomb Memorial Drive, Rochester, NY 14623, USA

${ }^{58}$ Current address: Department of Physics, Kyoto University, 606-8202 Kyoto, Japan. 
42 Department of Aeronautics and Astroautics, The University of Tokyo, Tokyo 113-8656, Japan

${ }^{43}$ Department of Aerospace Engineering, National Defense Academy, Yokosuka 239-8686, Japan

${ }^{44}$ Faculty of Intergrated Human Studies, Kyoto University, Kyoto 606-8501, Japan

45 Niigata University, Niigata 950-2181, Japan

46 Highfield, Southampton SO17 1BJ, UK

47 Department of Physics, Rikkyo University, Tokyo 171-8501, Japan

${ }^{4}$ Department of Information Systems, Osaka Institute of Technology, Hirakata 573-0196, Japan

49 Theoretical Astrophysics, Institute for Astronomy and Astrophysics, Eberhard Karls University of Tuebingen, Auf der Morgenstelle 10, 72076 Tuebingen, Germany

${ }^{50}$ Graduate School of Science, Nagoya University, Aichi 464-8601, Japan

51 Department of Management and Information Systems Science, Nagaoka University of Technology, Niigata 940-2188, Japan

52 Department of Electronics and Computer Science, Nihon University, Funabashi 274-8501, Japan

53 Department of Physics, Tokyo University of Science, Tokyo 162-8601, Japan

54 Graduate School of Science and Engineering/Physics, Tokyo Institute of Technology, Tokyo 152-8550, Japan

55 Tsukuba Space Center, Japan Aerospace Exploration Agency (JAXA), Ibaraki 305-8505, Japan

${ }^{56}$ Research Institute for Sustainable Humanosphere, Kyoto University, Kyoto 611-0011, Japan

57 Nakamura-minami Nerima, Tokyo 176-0025, Japan

E-mail: ando@granite.phys.s.u-tokyo.ac.jp

Received 31 October 2008, in final form 17 December 2008

Published 20 April 2009

Online at stacks.iop.org/CQG/26/094019

\begin{abstract}
DECIGO pathfinder (DPF) is a milestone satellite mission for DECIGO (DECihertz Interferometer Gravitational wave Observatory), which is a future space gravitational wave antenna. DECIGO is expected to provide fruitful insights into the universe, particularly about dark energy, the formation mechanism of supermassive black holes and the inflation of the universe. Since DECIGO will be an extremely challenging mission, which will be formed by three drag-free spacecraft with $1000 \mathrm{~km}$ separation, it is important to increase the technical feasibility of DECIGO before its planned launch in 2024. Thus, we are planning to launch two milestone missions: DPF and pre-DECIGO. In this paper, we review the conceptual design and current status of the first milestone mission, DPF.
\end{abstract}

PACS numbers: $04.30 . \mathrm{Tv}, 04.80 . \mathrm{Nn}$, 95.55.Ym, 95.85.Sz

\title{
1. Introduction
}

DECIGO, a DECi-hertz Interferometer Gravitational wave Observatory, is a space gravitational-wave antenna planned to be launched in 2024 [1, 2]. The purpose of DECIGO is to observe gravitational waves in the frequency band mainly between $0.1 \mathrm{~Hz}$ and $10 \mathrm{~Hz}$, and thus to open a new window of gravitational-wave astronomy. Since the observation band of DECIGO is between that of LISA [3] (around $1 \mathrm{mHz}$ band) and terrestrial detectors (around $100 \mathrm{~Hz}-\mathrm{a}$ few kHz band), such as Advanced LIGO [4], LCGT [5], Advanced VIRGO [6] and ET [7], it can be a follow-up of LISA and can also be a predictor for terrestrial detectors by observing binary inspiral sources. Moreover, since DECIGO's observation band is free from foreground noises caused by unresolved gravitational waves from many galactic binaries, it 


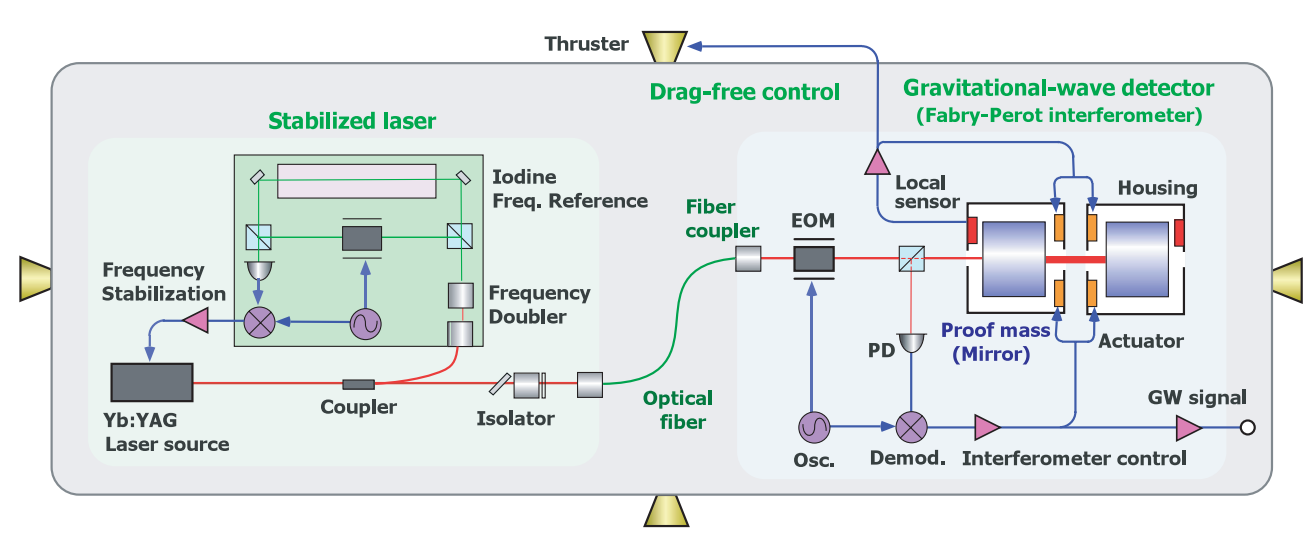

Figure 1. Conceptual design of the mission payload of the DECIGO pathfinder (DPF).

has a potential to observe stochastic background gravitational waves from the early universe. In the current design, DECIGO will be formed by three drag-free spacecraft that are separated by $1000 \mathrm{~km}$ from one another [8]. The gravitational-wave signals are detected by measuring their relative displacements with Fabry-Perot interferometers. The arm length was chosen so as to realize a finesse of ten with a $1 \mathrm{~m}$ diameter mirror and a laser beam with a $532 \mathrm{~nm}$ wavelength. The mass of the mirror is $100 \mathrm{~kg}$ and the laser power is $10 \mathrm{~W}$. Since DECIGO will be an extremely large mission both in its scale and required resources, it is significant to increase the technical feasibility before its launch. Thus, we have a roadmap to launch two milestone missions before DECIGO. DECIGO pathfinder (DPF) is the first milestone mission to test the key technologies with single spacecraft. Pre-DECIGO is supposed to detect gravitational waves with minimum specifications.

DPF will be a small satellite orbiting the Earth. The mission part of DPF is designed to be a prototype of DECIGO being comprised of a short Fabry-Perot cavity, a stabilized laser source and a drag-free control system (figure 1). DPF has, broadly speaking, two purposes: to make scientific observations of gravitational waves and the Earth gravity distribution, and to test key technologies for future space missions, such as DECIGO. In this paper, we review the conceptual design, scientific objectives and current status of DPF.

\section{Mission conceptual design}

A conceptual design of the DPF mission payload and an overview of the DPF satellite are shown in figures 1, 2 and table 1. DPF will be a small satellite with a weight of about $350 \mathrm{~kg}$, orbiting the Earth at an altitude of $500 \mathrm{~km}$. DPF will be launched by a next-generation solid propellant rocket, which is being developed as a successor of the M-V launch vehicle of JAXA (Japan Aerospace Exploration Agency). For stable power generation and temperature equilibrium of the satellite, DPF will have a circular Sun-synchronous dawn-to-dusk orbit and an Earth-synchronous attitude. Two proof masses inside the satellite will trail along in the same orbit. The attitude of the satellite will be passively stabilized by the gravity gradient of the Earth and actively controlled by a drag-free control system to cancel external disturbances. For this control, small mission thrusters will be used; momentum wheels will not be loaded so as to avoid their mechanical disturbances.

The DPF satellite will be formed by the combination of a bus module and a mission payload module. We are planning to use a standard bus system module under development in JAXA, which has a weight of about $200 \mathrm{~kg}$ and a size of $950 \times 950 \times 1100 \mathrm{~mm}$. This bus will 


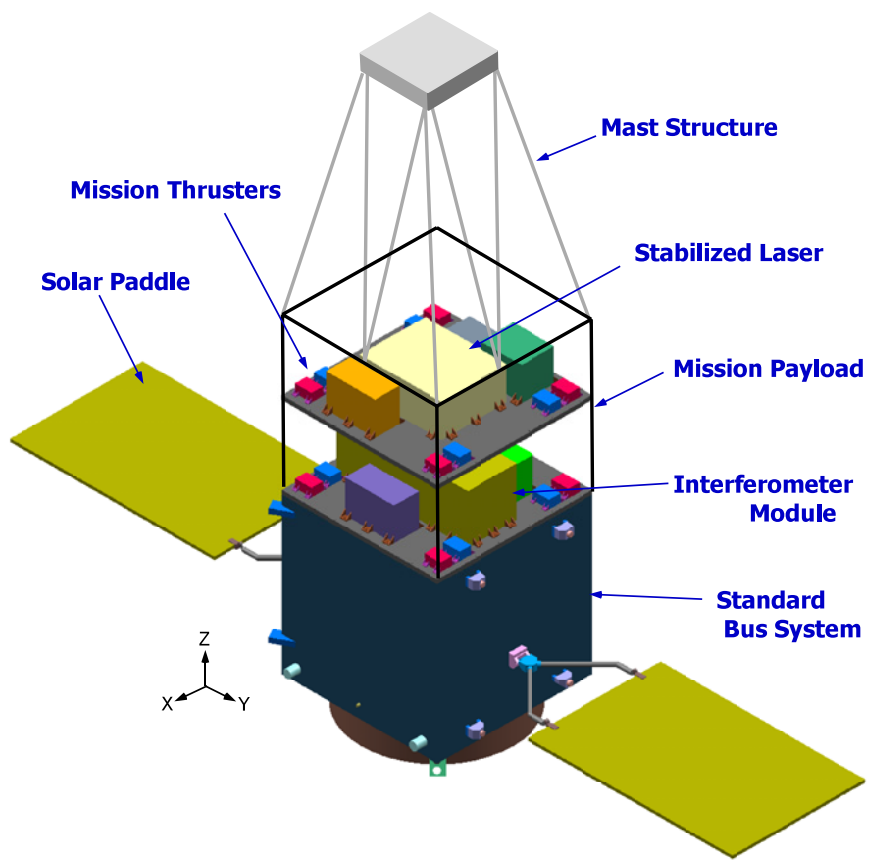

Figure 2. Overview of DPF. The satellite is comprised of a bus system part and a mission payload part.

provide a $940 \mathrm{~W}$ power with four solar-cell puddles and a 2 Mbps downlink telecommunication ability. A mission module will be attached on the upside of the bus module. The data processing system is based on a SpaceWire-based communication standard. The bus and mission modules are to be connected with power lines and SpaceWire communication lines, and cables for temperature controls.

The mission payload part of DPF will have a size of $950 \times 950 \times 1100 \mathrm{~mm}$, and a mast structure for gravity-gradient attitude stabilization will be attached at the top of the module. The mission part is designed to be a prototype of DECIGO, being comprised of a short Fabry-Perot cavity, a stabilized laser source and a drag-free control system (figure 1). The Fabry-Perot cavity is formed by two mirrors which act as free proof masses. Each mirror is to be placed inside a module called housing. The housing will have electrostatic-type local sensors and actuators on its frame, which are to be used to monitor and control the relative motion between the mirror and the frame in all degrees of freedom. In addition, the housing will have a function of a launch lock, which will clump the mirror at the launch of the satellite and release it in the orbit with a small initial velocity. The cavity will have a baseline length of about $30 \mathrm{~cm}$ and a finesse of about 100. The length change in the Fabry-Perot cavity, which would be caused by gravitational waves or external disturbances, is to be measured by means of a stabilized laser beam. The PDH scheme will be used for the signal extraction and control of the Fabry-Perot cavity length [9]. In DPF, we will use a Yb:YAG laser source in which the frequency is stabilized using saturated absorption spectroscopy of iodine molecules. The requirement for the frequency stabilization is $0.5 \mathrm{~Hz} / \mathrm{Hz}^{1 / 2}$. The laser source will have an output power of $100 \mathrm{~mW}$ at a wavelength of $1030 \mathrm{~nm}$. The drag-free control of the satellite will work as a shield against external forces caused by solar radiation and drag by residual 
Table 1. Summary of DECIGO Pathfinder mission.

\begin{tabular}{|c|c|c|}
\hline Item & Feature & Comment \\
\hline \multicolumn{3}{|l|}{ Mission and satellite } \\
\hline Launch target & FY 2012 & 1 year mission lifetime \\
\hline Launcher & $\begin{array}{l}\text { Solid propellant rocket } \\
\text { (M-V follow-on) }\end{array}$ & $\begin{array}{l}\text { Require the PBS } \\
\text { (post-boost stage) }\end{array}$ \\
\hline Orbit & $\begin{array}{l}\text { Sun-synchronous circular orbit } \\
\text { Altitude } 500 \mathrm{~km}\end{array}$ & $\begin{array}{l}\text { Dawn-to-dusk orbit } \\
\text { Inclination } 97.4 \mathrm{deg}\end{array}$ \\
\hline \multirow[t]{2}{*}{ Attitude } & Sun- and Earth-synchronous & $\begin{array}{l}\text { Gravity-gradient stabilization } \\
\text { and } 3 \text {-axis active control }\end{array}$ \\
\hline & Drag-free control in all DOF & During mission operation \\
\hline Size & $950 \times 950 \times 2000 \mathrm{~mm}$ & Excluding the mast structure \\
\hline Weight & $350 \mathrm{~kg}$ & \\
\hline Power consumption & Max $600 \mathrm{~W}$ & \\
\hline \multicolumn{3}{|l|}{ Mission payload } \\
\hline \multirow[t]{2}{*}{ GW detector } & $\begin{array}{l}\text { Fabry-Perot interferometer } \\
\text { Sensitivity } 2 \times 10^{-15} \mathrm{~Hz}^{-1 / 2}\end{array}$ & Baseline length $30 \mathrm{~cm}$ \\
\hline & Proof mass $\sim 1 \mathrm{~kg} \times 2$ & Require a launch-lock system \\
\hline Earth-gravity detector & $\begin{array}{l}6 \times 2 \text { laser sensors (MI type) } \\
\text { Semiconductor laser } 40 \mathrm{~mW}\end{array}$ & $\begin{array}{l}\text { Attached to proof mass housings } \\
\text { Introduced via optical fibers }\end{array}$ \\
\hline Stabilized laser & $\begin{array}{l}25 \mathrm{~mW} \text { on the interferometer } \\
\text { Frequency stability } 0.5 \mathrm{~Hz} / \mathrm{Hz}^{1 / 2}\end{array}$ & $\begin{array}{l}\text { Output with an optical fiber } \\
\mathrm{I}_{2} \text {-saturated absorption spectroscopy }\end{array}$ \\
\hline Drag-free control & $\begin{array}{l}\text { Better than solar radiation noise } \\
12 \text { mission thrusters }\end{array}$ & Force $100 \mu \mathrm{N}$, Noise $0.1 \mu \mathrm{N} / \mathrm{Hz}^{1 / 2}$ \\
\hline $\begin{array}{l}\text { Size } \\
\text { Weight } \\
\text { Power consumption }\end{array}$ & $\begin{array}{l}950 \times 950 \times 900 \mathrm{~mm} \\
150 \mathrm{~kg} \\
120 \mathrm{~W}\end{array}$ & Excluding the mast structure \\
\hline \multicolumn{3}{|l|}{ Standard bus system } \\
\hline Data processing & $\begin{array}{l}\text { CPU HR5000, } 33 \mathrm{MHz} \\
\text { Data recorder } 2 \text { GByte } \\
\text { Down link } 2 \mathrm{Mbps}\end{array}$ & SpaceWire-based processing system \\
\hline Power supply & $\begin{array}{l}2 \text { solar puddles } \times 2 \text { sides } \\
\text { battery } 50 \mathrm{Ah}\end{array}$ & $\begin{array}{l}\text { Power generation } 940 \mathrm{~W} \\
50 \mathrm{~V}, 150 \mathrm{~W} \text { to the mission payload }\end{array}$ \\
\hline Attitude control & $\begin{array}{l}3 \text {-axis control } \\
3 \mathrm{~N} \text { thrusters } \times 4\end{array}$ & Initial operation and fail-safe \\
\hline $\begin{array}{l}\text { Size } \\
\text { weight }\end{array}$ & $\begin{array}{l}950 \times 950 \times 1100 \mathrm{~mm} \\
200 \mathrm{~kg}\end{array}$ & Solar paddles folded \\
\hline
\end{tabular}

atmosphere. Drag-free control will be realized by measuring the relative fluctuations between the mirrors and the satellite and basically feeding these signals back to the satellite position using low-noise mission thrusters. The satellite motion will be controlled in all degrees of freedom with a bandwidth of about $10 \mathrm{~Hz}$. DPF has two test mass mirrors inside it; roughly, the common motion signals of two test masses will be used for the drag-free control of the satellite, and the differential motion signals will be fed back to the test masses so as to stabilize the Fabry-Perot cavity. 

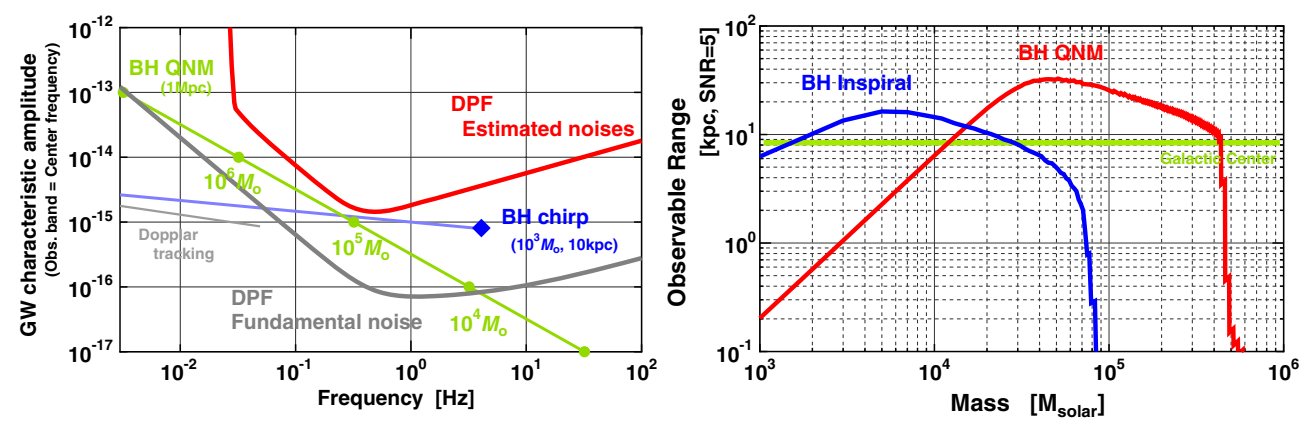

Figure 3. Sensitivity (left) and observable range (right) of DPF. The observable range of DPF for intermediate-mass black holes will cover the center of our galaxy.

\section{Scientific objectives of DPF}

\subsection{Scientific observation}

DPF will have a $30 \mathrm{~cm}$ Fabry-Perot cavity formed by two proof-mass mirrors. Each proof mass will be kept inside the spacecraft untouched to avoid external disturbances and to be sensitive to gravitational forces, such as gravitational waves and Earth's gravity distributions.

Gravitational waves would be detected as tidal-force fluctuations on two proof masses; the length change in the Fabry-Perot cavity is to be measured by a laser interferometer. The DPF interferometer will have a sensitivity limit of about $h \sim 10^{-15}$ at around the frequency band of 0.1-1 Hz. At this frequency band, it is expected that gravitational waves from intermediatemass blackhole inspirals and quasi-normal mode of massive blackholes will be radiated. The observable ranges of DPF for these phenomena are estimated as a function of the BH masses with a detection threshold of a signal-to-noise ratio of 5 (figure 3 ). DPF has a potential to detect gravitational-wave signals, if there is a black-hole inspiral event with $10^{3}-10^{4} M_{\odot}$, or a ringdown event of a quasi-normal mode for $10^{4}-4 \times 10^{5} M_{\odot}$ black hole at the center of our galaxy. Although the probability to have such events is considered to be rare, data obtained by DPF observations will have importance, because this observation band is difficult to access by ground-based gravitational-wave detectors and other space-based detection methods.

Observation of the gravity of the Earth is another scientific objective of DPF. Since the proof masses orbit the Earth almost freely, gravity distributions of the Earth would be observed from the trajectories of the proof masses. In order to cancel the drag force by air and solar radiation, the relative displacements between the proof masses and the satellite frame are to be measured by small Michelson-interferometer-type laser sensors with an acceleration sensitivity of $10^{-11} \mathrm{~m} \mathrm{~s}^{-2}$. Several satellites to observe Earth's gravity have been launched so far [10,11], and one with better sensitivity will be launched soon [12]. The characteristic feature of DPF is to make the sensitive accelerometer in a sufficiently small package. This module would be easier to be loaded in a future satellite network for high-resolution and real-time monitoring of Earth's gravity.

\subsection{Development of key technologies for future missions}

The key technologies tested in DPF will be the following: (1) low-noise operation and observation with a Fabry-Perot interferometer in space, (2) operation of a laser stabilization system in a space environment and (3) demonstration of a drag-free control system. All of 
these technologies are critical for the realization of DECIGO and are also useful for other future missions.

The main Fabry-Perot interferometer in DPF with a baseline length of $30 \mathrm{~cm}$ is designed as a prototype of $1000 \mathrm{~km}$ arm cavity of DECIGO. Although measurement and operation with such an interferometer is a well-established technique in a ground-based environment, there is no example of a Fabry-Perot cavity formed by free floating mirrors in a space environment. In a Fabry-Perot configuration, we can expect better sensitivity than that of a Mach-Zender interferometer, which is used in LPF [13], because the distance between two floating test masses is directly measured in a Fabry-Perot interferometer. However, the control configuration to keep the proof mass mirrors inside the satellite and, at the same time, to operate the interferometer stably will be more complex. The demonstration of Fabry-Perot interferometer operation in DPF will provide a new possibility for precise measurements in a space environment.

In DPF, the frequency noise of the laser source is stabilized using saturated absorption spectroscopy of iodine molecules, targeting at a stability of $0.5 \mathrm{~Hz} / \mathrm{Hz}^{1 / 2}$ in the $0.1 \mathrm{~Hz}$ frequency band. Frequency stabilization of a laser source has been well studied for groundbased gravitational-wave detectors and in other fields, such as precise metrology, spectroscopy, optical communications and so on. On the other hand, there are few experiments in a space environment. However, recently, laser sources with high frequency stability have come to be required in space missions [14]. For example, the Earth Explorer Atmospheric Dynamics Mission (ADM-Aeolus) requires a $25 \mathrm{kHz} / \mathrm{Hz}^{1 / 2}$ frequency stabilized laser and ACES (Atomic Clock Ensemble in Space) on the ISS (International Space Station) requires $100 \mathrm{~Hz} / \mathrm{Hz}^{1 / 2}$ frequency stability. LISA requires $30 \mathrm{~Hz} / \mathrm{Hz}^{1 / 2}$ frequency stability. Compared to these missions, the stability of $0.5 \mathrm{~Hz} / \mathrm{Hz}^{1 / 2}$ in DPF will be a significant step toward showing the potential of a stabilized laser, while it will be rather challenging in a space environment.

Drag-free control is a scheme used to avoid the effect of external forces on a satellite, such as drag by a residual atmosphere along the orbit and radiation pressure of the Sun. Drag-free control is realized by controlling the position of a satellite to follow the motion of a proof mass, which is placed inside the satellite and is shielded from external forces by the satellite. Drag-free control of a satellite was realized by the TRIAD-I satellite in 1972 for the first time [16] and several follow-on satellites for investigating a navigation system. Recently, drag-free control has also been realized by the Gravity Probe-B satellite for tests of the general theory of relativity [17]. LPF will demonstrate drag-free control at the Lagrange 1 (L1) point between the Earth and the Sun, at which the gravitational environment is stable. On the other hand, DPF will demonstrate it in an Earth orbit, with the help of passive attitude stabilization using gravity gradient of the Earth so as to reduce the effect of artificial mechanical fluctuations by attitude-control actuators, such as momentum wheels. This will be a new scheme to realize a drag-free satellite.

\section{Current status of DPF}

Currently, DPF has been selected as one of the candidates of small satellite missions of JAXA. JAXA has a project to launch at least three small satellites in five years from 2011, using standard bus systems. The first mission of the three missions has already been decided to be TOPS, which is for the observation of inferior planets. TOPS will be launched in FY 2011. DPF is now one of several high-ranked mission candidates for the second or third missions, and will be launched in FY 2012 in the best and earliest case.

Research and development are underway with the support of JAXA, mainly concerning a mission study including satellite design and drag-free control topology and on testing key 
devices, such as a housing system for a proof mass, a stabilized laser, and thrusters. In addition, a small demonstration module, named SWIM (SpaceWire Interface demonstration Module), has been developed and will be launched in FY2008 in JAXA's technology demonstration satellite; the flight module has already been fixed to the satellite for a series of total tests. The SWIM contains a space-qualified data processor and recorder with the SpaceWire interface, and a tiny gravitational-wave detector module with a size of $160 \times 80 \times 80 \mathrm{~mm}$. SWIM will provide heritages for DPF on a SpaceWire-based data processing system and on sensing and control of proof masses in a space environment.

\section{Conclusion}

DECIGO will open a new window for gravitational-wave astronomy, and the DECIGO pathfinder (DPF) will be the first significant milestone mission to test the key technologies for DECIGO. Moreover, DPF will provide new scientific knowledge on gravitational-wave observations, on the gravity of Earth, on precise measurements with an interferometer, on laser stabilization in space, and on drag-free control of a spacecraft. DPF has been selected as one of the candidates of small satellite missions of JAXA, and research and development for key components are underway. We are hoping to launch DPF in FY 2012 in the best case.

\section{Acknowledgments}

This research was supported by the Japan Aerospace Exploration Agency (JAXA), and by the Japan Society for the Promotion of Science (JSPS), Grant-in-Aid for Scientific Research.

\section{References}

[1] Seto N, Kawamura S and Nakamura T 2001 Phys. Rev. Lett. 87221103

[2] Kawamura S et al 2006 Class. Quantum Grav. 23 S125

[3] LISA 2000 LISA: System and Technology Study Report ESA document ESA-SCI 2000

[4] Fritschel P 2002 Second generation instruments for the Laser Interferometer Gravitational Wave Observatory (LIGO) Gravitational-Wave Detection (4856-39): Proc. SPIE Meeting ed P Saulson and M Cruise (Waikoloa, Hawaii) pp 282-91

[5] Kuroda K et al 2002 Class. Quantum Grav. 191237

[6] The Virgo Collaboration 2007 Advanced Virgo Conceptual Design Virgo Report VIR 042A 07, http://wwwcascina.virgo.infn.it/advirgo/docs/AdV_Design.pdf

[7] http://www.et-gw.eu

[8] Sato S 2009 J. Phys. Conf. Ser. 154012040

[9] Drever R W P, Hall J L, Kowalski F V, Hough J, Ford G M, Munley A J and Ward H 1983 Appl. Phys. B 3197

[10] Champ mission Web Page, http://op.gfz-potsdam.de/champ/index_CHAMP.html

[11] GRACE mission Web Page, http://www.csr.utexas.edu/grace

[12] GOCE mission Web Page, http://www.esa.int/esaLP/LPgoce.html

[13] LISA Pathfinder Web Page, http://sci.esa.int/science-e/www/area/index.cfm?fareaid=40

[14] Nagano S 2008 in the mission proposal of DPF (in Japanese)

[15] Tsuda $\mathrm{T}$ internal document

[16] TRIAD I 1974 AIAA J. Spacecraft 11 pp 637-44

[17] Gravity Probe B Web Page, http://www.gravityprobeb.com/ 